\title{
Aplicación de un SIGP como plataforma multiactoral para la visualización y análisis de percepciones de problemas ambientales en San Cristóbal de Las Casas, Chiapas, México
}

\author{
GIS application for the visualization and analysis of \\ perceptions of environmental problems in \\ San Cristóbal de Las Casas, Chiapas, Mexico
}

\section{Gabriela Mariana Fenner Sánchez*}

\section{Resumen}

Situada en la ciudad de San Cristóbal de Las Casas, Chiapas, la investigación tuvo el objetivo de desarrollar un Sistema de Información Geográfica Participativo, que permitiera conocer qué problemas ambientales perciben diferentes tipos de actores en la ciudad, desde un enfoque territorial y de la geografía humana. Se diseñó por tanto una metodología mixta que permitiera trabajar con información cualitativa, y que a la vez fuera pertinente para la escala de la ciudad. Así, a través de cartografía participativa se trabajó con ciudadanía en general, organizaciones sociales y sector gubernamental municipal encargado de temas territoriales o ambientales. Los resultados fueron integrados en un SIGP por medio del cual se generaron contrastes y complementariedades entre percepciones de los diferentes actores, así como con información cartográfica complementaria, como planes de ordenamiento territorial. La metodología mostró su utilidad para identificar zonas prioritarias, temas clave y potenciales sinergias entre actores para una mejor incidencia en materia ambiental.

* Centro de Estudios sobre México y Centroamérica (CESMECA-UNICACH), México, correo electrónico: proyecto.mesoamerica@gmail.com. ORCID: https://orcid.org/0000-0003-0903-4293. 
Palabras clave: cartografia participativa, SIGP $(P)$, percepciones, problemas ambientales urbanos, Chiapas.

\begin{abstract}
Located in the city of San Cristóbal de Las Casas, Chiapas, the study aimed at developing a Participatory Geographic Information System (PGIS), which would reveal how environmental problems are perceived by different stakeholders in the city, from a territorial and human geography perspective. A mixed methodology was therefore designed to work with qualitative information, but at the same time relevant to the scale of the city. Thus, through participatory mapping, the work was carried out with the general public, social organizations and the municipal government sector in charge of territorial or environmental issues. The results were integrated into a PGIS through which contrasts and complementarities were generated among the perceptions of the different stakeholders, as well as with complementary cartographic information, such as land use plans. The methodology demonstrated its usefulness in identifying priority areas, key issues and potential synergies between stakeholders for a better impact on environmental matters.

Key words: participatory mapping, (P) PGIS, perceptions, urban environmental problems, Chiapas.
\end{abstract}

\title{
Planteamiento del problema
}

Debido a las implicaciones territoriales que conlleva el manejo ambiental, se han utilizado metodologías distintas en el ámbito rural y urbano. Para el primero, ha habido mayor exploración de aquellas denominadas participativas, por corresponder más adecuadamente a las formas de organización social existentes en dicho ámbito, mientras que, en las ciudades, por la propia dimensión de sus problemas, composición social y ritmo de vida, generalmente se prefieren metodologías indirectas, o bien, si se pretende contar con la opinión de las y los habitantes, se utilizan métodos rápidos aleatorios como las encuestas. Las ciudades medias, sin embargo, representan cierto intersticio entre estos dos mundos de análisis y de intervención, tanto por el tipo de problemas que presentan, como por la composición social que permitiría un esfuerzo coordinado entre actores. La presente investigación, desde un pensamiento situado, busca contribuir en la construcción de metodologías que puedan ser útiles en estos contextos en material ambiental y desde un enfoque territorial.

La investigación se desarrolló en San Cristóbal de Las Casas (SCLC), ciudad media, cabecera del municipio del mismo nombre y que está ubicada a $2130 \mathrm{msnm}^{1}$. Esta ciudad para el año 2010 contaba con una población de 158027 habitantes

1 Esta altitud corresponde al parque central, la ciudad presenta diferentes altitudes, llegando hasta los $2300 \mathrm{msnm}$ aproximadamente. 
(INEGI, 2010), ${ }^{2}$ registrando el municipio en 2015 un crecimiento anual de 2.7\% (INEGI, 2015). A pesar de su importancia social, política y económica en el estado de Chiapas, se trata de una ciudad sin industrias significativas, que ha basado su economía en la renta del suelo y de la fuerza de trabajo. En la actualidad, su principal actividad económica es el turismo, estando las demás actividades supeditadas a él.

El crecimiento urbano acelerado, sobre todo en los últimos 40 años, ha incrementado igualmente las necesidades de recursos naturales utilizados. Los problemas ambientales identificados en las últimas décadas tienen que ver precisamente con el agotamiento y contaminación de recursos, como es el caso de los ríos contaminados, los humedales secados por la construcción, la destrucción de cerros por bancos de arena, la contaminación atmosférica, la incapacidad de tratar los residuos, etc. A pesar de que diferentes instancias tienen jurisdicción sobre estos problemas, pocos son los avances que se han logrado. A la par de estas instancias existen también organizaciones de la sociedad civil, escuelas, universidades y otras iniciativas privadas que han tomado acciones para el "cuidado del medio ambiente"; sin embargo, la mayoría de ellas son aisladas, sectoriales y puntuales.

A partir del análisis de información documental, además de la experiencia de la autora al vivir en esta ciudad, se llegó a formular el supuesto de que esta situación es así porque hay una falta de autorreconocimiento, como habitantes de la ciudad, del papel que se tiene en la conformación del propio territorio y sus implicaciones ambientales, además de que no existen suficientes espacios de participación ciudadana directa y, como consecuencia, prevalece una invisibilización de los saberes locales, así como de las prioridades y de las pertenencias, que podrían ser punto de partida para alimentar, fomentar o hacer efectivas las políticas públicas encaminadas a prevenir y/o enfrentar los problemas ambientales. Sin obviar tampoco que detrás de muchos de los problemas ambientales existen intereses económicos o políticos no manifiestos, que obstaculizan la transformación de esa realidad negativa. A ello se suma el hecho de que entre los diferentes actores sociales que se involucran en el tema no existe un solo discurso y, por lo tanto, las propuestas hechas por unos generan a veces conflictos con otros, mermando su factibilidad.

Así, desde el enfoque de la geografía humana y humanista, que toma en cuenta la dimensión subjetiva del habitar, sin descuidar, ni las relaciones de poder, ni la materialidad del espacio, se pensó en la necesidad de una posible plataforma de diálogo para actores distintos, por lo que se planteó construir un sistema de información geográfica participativo (SIGP), en el que el lenguaje cartográfico estandarizado permitiera ese punto de encuentro entre diferentes actores (McCall, 2011). La apuesta por la cartografía se sostiene, además, en una comprensión crítica de la misma, mediante

2 En México, los censos poblacionales se llevan a cabo cada 10 años, de ahí que aún no se cuente con una cifra más actualizada. 
la cual el mapa se revela como instrumento de poder, como medio de comunicación, como signo y como discurso (Harley, 2005). A lo que Kitchin \& Dodge (2007) añaden que es el mismo contexto y los actores quienes le otorgan el poder a los mapas, siendo éstos pues siempre emergentes y contingentes.

A partir de la literatura revisada, se decidió combinar principios epistemológicos de los SIGP (y SIGPP) y de la información geográfica voluntaria (IGV). De los primeros se retomó el énfasis en los procesos mismos, en los que los talleres se convierten en los momentos más importantes, donde sucede el diálogo, el aprendizaje, y el empoderamiento (McCall, 2004, 2011; Rambaldi, Chambers et al., 2006); mientras que de la segunda se exploran las potencialidades de ejercicios rápidos en los cuales se pretende abarcar más participantes, tomando entonces el resultado mayor importancia que el proceso (Goodchild, 2007; Brown, 2016; Verplanke et al., 2016). Así, se diseñaron dos métodos: sesiones callejeras de mapeo colaborativo para trabajar con ciudadanía en general, y talleres de mapeo colectivo para trabajar con organizaciones sociales y con sector gubernamental municipal responsable de las políticas ambientales y/o territoriales. Los resultados alimentaron un SIG que fue procesado por medio del software QGIS 2.14 (Essen), generando cartografía analítica que fue complementada con información fisiográfica, así como con datos provenientes del Plan de Ordenamiento Ecológico Territorial del municipio (SEMARNAT/SEMAHN, 2013).

El objetivo del presente trabajo no es tanto mostrar los mapas resultado, sino principalmente la ruta metodológica y la reflexión epistemológica detrás de ella, así como los aprendizajes obtenidos para dar continuidad y hacer replicable la metodología.

\section{Diseño metodológico}

La metodología encuentra así, parte de su justificación en los fundamentos ontológicos de los SIGP en cuanto a dar voz a quienes no tienen voz, pero a la vez utiliza métodos (técnicas y tiempos) que son más cercanos a una consulta pública de tipo SIGPP, cuya pregunta básica es: “¿Qué de las posesiones individuales de conocimiento y entendimiento del lugar pueden contribuir sustancialmente al proceso de planeación?"4 (Brown, 2012: 7). Lo cual, parte de asumir que existe un conocimiento espacial local acumulado por las y los habitantes acerca de su entorno geográfico, basado en sus experiencias cotidianas; "este conocimiento es la base para recomendaciones o acciones, que la comunidad, ya sea en su totalidad o individualmente, ha adoptado respecto a su ambiente" (Rodríguez, 2003: 157).

3 Sistemas de Información Geográfica Pública Participativa es uno de los nombres que se le ha dado a los SIG que integran participación de población no experta. Hay quienes atribuyen a esta un uso más desde las instancias oficiales para legitimar programas, en comparación con los SIGP que se realizan desde iniciativas no gubernamentales en contextos reivindicativos.

4 Traducción propia. 
En este caso, busqué además contrastar el conocimiento espacial local de diferentes tipos de actores a cierto nivel de conocimiento experto. Ya que, siguiendo a Pfeffer et al.,

el proceso participativo es necesario para visibilizar varios tipos de conocimiento (...), así como para incluir actores que usualmente están excluidos (...) El SIG que vincula el conocimiento a un área visualiza los diversos tipos de conocimiento y hace más explícitos los intercambios entre prioridades, conflictos y $\operatorname{sinergias}^{5}$ (Pfeffer, Baud, Denis, Scott \& Sydenstricker-Neto, 2010).

Así, tomando en cuenta la advertencia que hace McCall (2004) de que la intensidad de la participación debe, no tanto ser la máxima, como sí, la apropiada a las tareas, competencias y relaciones específicas entre los actores (p. 4), esta metodología utilizó diferentes métodos de mapeo según las condiciones y posibilidades de los diferentes actores; promoviendo la participación con el objetivo de "generar vínculos" (McCall, 2004: 6), y asumiéndola como "mecanismo indispensable de socialización de conocimientos y prácticas [que se nutre de] la cotidianidad y de una noción de territorio que involucra a las y los habitantes" (ENDA América Latina-Colombia, 2003: 1).

A fin de controlar la efectividad de la metodología para funcionar como dispositivo de diálogo y acción para el quehacer ambiental, se decidió tomar en cuenta los siguientes criterios: ${ }^{6}$

- Validez: ¿qué tanto responde la metodología a las necesidades del problema planteado?

- Representatividad: ¿qué tanto quienes están siendo tomados en cuenta, representan a la totalidad de los actores implicados en diferentes formas en la problemática abordada?

- Practicidad de su aplicación (factibilidad): ¿qué tan posible es llevar a cabo el proceso; en términos de tiempo, de costos, de logística?

- Aceptación: ¿cómo responden las y los participantes a las dinámicas?

\section{Fase 1. Identificación de actores y lugares clave}

Según el recuento que hace Ghose (2017) acerca de los SIGPP, lo "público incluye no solamente a las comunidades marginalizadas, sino también a un amplio rango de actores y a una multiplicidad de instituciones" (p. 2) y retoma de Schlossberg y Shuford (2005) que éstos pueden ser: a) los afectados por las decisiones de algún programa, b) aquellos que puedan aportar conocimiento o información importante 
para las decisiones del programa, y c) aquellos que tienen el poder de influenciar y/o de afectar la implementación o decisiones de un programa. Con esto presente, a partir de información hemerográfica, documentos del archivo municipal, el Plan de Ordenamiento Ecológico Territorial, así como de anteriores investigaciones, se identificaron seis tipos de actores o grupos; a saber: ciudadanía en general, ciudadanía organizada (organizaciones vecinales), organizaciones no gubernamentales, sector académico, sector empresarial, y ayuntamiento municipal (en sus áreas correspondientes a la gestión territorial y/o ambiental). De entre los cuales, razones logísticas llevaron a elegir trabajar con organizaciones sociales, ayuntamiento y ciudadanía en general; tomando en cuenta que "la elección de herramientas para facilitar una participación efectiva deberá estar dictada no solamente por el nivel de involucramiento público pretendido, sino por las restricciones de la esfera del problema y por las características de los participantes" (Jankowsky, 2009: 1968).

Los lugares para los mapeos callejeros se eligieron a partir de realizar recorridos para hacer testimonio visual de algunos de los problemas ambientales, lo cual fue complementado con la literatura; además de cuidar el tener una cobertura lo más equitativa posible de las diferentes zonas de la ciudad; pues si bien el muestreo no sería representativo en términos estadísticos, sí era posible procurar tener información de colonias y/o barrios con diferentes características, por ejemplo en cuanto a su conformación social, antigüedad, cercanía a zonas identificadas en la literatura como problemáticas en términos ambientales, etc. Además se tomó en cuenta la posibilidad práctica de contar con un espacio de conglomeración como son los parques y canchas.

\section{Fase 2. Diseño e implementación de sesiones y talleres de mapeo}

El propósito de los talleres y las sesiones callejeras era identificar qué percibían los diferentes actores como problemas ambientales en la ciudad. Desde una perspectiva territorial se trataba, además, de reflejar el conocimiento local, las escalas de apropiación territorial desde la cotidianidad, las relaciones de poder implicadas en los asuntos ambientales (y el nivel de empoderamiento ciudadano), la perspectiva de ciudad; así como la visión temporal (histórica) de la misma. Puesto que como bien señala Carver (2001) los problemas ambientales tienen una fuerte componente espacial, por lo que utilizar el espacio para situar las decisiones respecto a ellos es altamente benéfico para organizar, tanto nuestra visión del problema como nuestro compromiso con él.

Esta etapa se dividió en dos tipos de trabajo distinto: fundamentadas en los principios de la IGV se realizaron las sesiones de mapeo callejero con población aleatoria (ciudadanía en general); mientras que, basándose en el mapeo colectivo, se diseñaron talleres que fueron implementados con las organizaciones sociales y el sector gubernamental. Para subrayar esta diferencia cabe aquí recordar que Verplanke et al., (2016), en su intento por nombrar las principales diferencias y similitudes entre los 
SIGP y la IGV, mencionaban que, ya que los SIGP involucran a comunidades locales en la creación de información espacial para alimentar un SIG, pero a la vez en la toma de decisiones respecto a esa información, puede decirse que uno de sus principales propósitos está directamente relacionado con propiciar el entendimiento propio y la conciencia sobre el territorio que se habita. En su lugar, la IGV pretende "crear, validar, analizar y difundir datos geográficos contribuidos voluntariamente por personas a lo individual [...por lo que] está basada más en contribución y comunicación de información, que en participación" (Verplanke et al., 2016: 3).

Los hitos trabajados, tanto en sesiones como en talleres eran casi los mismos, con excepción de las proyecciones temporales que pudieron trabajarse únicamente en los talleres. A continuación, se detallan los métodos empleados.

\section{Sesiones de mapeo callejero con población local}

El ejercicio consistió en colgar cuatro mapas con la traza urbana de la ciudad con los nombres de las calles, de dimensiones $1.10 \mathrm{~m}$ x $90 \mathrm{~cm}$, los cuales estaban cubiertos de plástico y tenían cada uno un título correspondiente a lo que se pedía mapear (Tabla 1).

Tabla 1

Dinámica usada en mapeos callejeros

\begin{tabular}{|c|c|c|c|c|}
\hline & $\begin{array}{c}\text { Mapal. } \\
\text { Problemas } \\
\text { ambientales }\end{array}$ & $\begin{array}{c}\text { Mapa } 2 . \\
\text { Causas de esos } \\
\text { problemas }\end{array}$ & $\begin{array}{c}\text { Mapa } 3 \text {. } \\
\text { ¿Quién debel puede ha- } \\
\text { cer qué para } \\
\text { solucionarlos? }\end{array}$ & $\begin{array}{c}\text { Mapa } 4 . \\
\text { ¿Qué había y ya no } \\
\text { hay? }\end{array}$ \\
\hline$\frac{0}{\frac{0}{0}}$ & $\begin{array}{l}\text { Pegar pequeños } \\
\text { papeles adhesivos } \\
\text { en el lugar donde se } \\
\text { ubica la } \\
\text { problemática } \\
\text { escribiendo cuál es }\end{array}$ & $\begin{array}{l}\text { Pegar } \\
\text { pequeños } \\
\text { papeles } \\
\text { adhesivos en el } \\
\text { lugar donde se } \\
\text { ubica la } \\
\text { problemática } \\
\text { escribiendo la } \\
\text { causa }\end{array}$ & $\begin{array}{l}\text { Pegar pequeños } \\
\text { papeles adhesivos en } \\
\text { el lugar donde se } \\
\text { ubica la } \\
\text { problemática } \\
\text { escribiendo qué } \\
\text { debería hacer el o } \\
\text { los actores } \\
\text { considerados } \\
\text { responsables }\end{array}$ & $\begin{array}{l}\text { Dibujo libre sobre } \\
\text { el mapa } \\
\text { plastificado con } \\
\text { marcadores } \\
\text { permanentes. } \\
\text { También se podía } \\
\text { escribir y colocar } \\
\text { anotaciones al } \\
\text { margen para } \\
\text { situaciones } \\
\text { generales }\end{array}$ \\
\hline $\begin{array}{l}n \\
0 \\
0 \\
0 \\
\text { के }\end{array}$ & $\begin{array}{l}\text { Categorización según } \\
\text { prioridad (urgencia o } \\
\text { importancia) a través } \\
\text { de sistema de colores } \\
\text { tipo semáforo (rojo, } \\
\text { amarillo, verde) }\end{array}$ & Ninguno & $\begin{array}{l}\text { Tipo de actor; } \\
\text { gubernamental o no } \\
\text { gubernamental, con } \\
\text { un color para cada } \\
\text { uno }\end{array}$ & $\begin{array}{l}\text { Ninguno (incluso } \\
\text { no se restringía al } \\
\text { tema ambiental) }\end{array}$ \\
\hline
\end{tabular}

Fuente: elaboración propia. 
Éstos se colocaron, siguiendo las sugerencias del método "puesto de mapeo" (Risler y Ares, 2013), en lugares públicos, a donde se invitaba a las personas que pasaban y mostraban algún interés, a participar, bajo la dinámica que se explica en la Tabla 1. Las sesiones duraron alrededor de dos horas.

\section{Talleres con las organizaciones sociales y las instancias gubernamentales}

En sesiones separadas, con cada organización y con integrantes del ayuntamiento municipal se realizaron talleres de cartografía social utilizando la metodología de "mesa rotativa de mapeo". Este método consiste en dividir el mapa, en este caso, de la ciudad de SCLC, en cuatro cuadrantes. Cada persona o grupo va trabajando por ronda o pregunta en cada uno de los cuadrantes, rotando después al siguiente, de tal manera que la información de cada cuadrante se va complementando con la participación de todas y todos (Risler y Ares, 2013: 26).

En ambos casos, la identificación y caracterización de los problemas siguió la dinámica expuesta en la Tabla 2. Como podrá observarse, en estos talleres, algunos aspectos se trabajaron directamente sobe el mapa y otros por medio de una matriz en la que se indicaba: 1) problema ambiental identificado, 2) causas, 3) actores responsables de solucionarlo y 4) acciones para solucionarlo.

Finalmente, ya uniendo los cuadrantes y completando el mapa, se les pidió generar, por medio de dos transparencias, sendos mapas más, en los que se mostraran escenarios tendenciales a 20 años (una generación), a partir de la información recabada: uno que mostrara cómo se vería la ciudad si se siguiera actuando como se ha hecho hasta la fecha; y otro donde se mostrara cómo sería si se implementaran las medidas sugeridas por los propios actores para solucionar los problemas ambientales por ellos mismos percibidos. Esta última propuesta de crear escenarios responde a asumir que

el manejo del lenguaje gráfico y el sentido colectivo del ejercicio permite complementar nociones y construir consensos frente a una realidad vivida y una realidad deseada en la que se visibilizan las apuestas individuales y de grupo, a la vez que estas acciones y pensamientos cualifican su sentido político, proyectando acciones desde el conocimiento del territorio en su conjunto y el reconocimiento de cada actor en ese territorio (ENDA América Latina-Colombia, 2003: 2).

El método de representación fue el dibujo libre, por lo que podían señalarse zonas, lugares puntuales, escribir textos, etc.

Cabe señalar que, mientras se hacían los mapeos también se tomaba nota acerca de los comentarios de las y los participantes, su actitud y respuesta ante los ejercicios planteados, entre otros aspectos que forman parte de la creación cartográfica participativa. 
Tabla 2

Dinámica usada en talleres de mapeo

\begin{tabular}{|c|c|c|c|c|c|c|}
\hline $\begin{array}{l}\text { Variable } \\
\text { Método }\end{array}$ & $\begin{array}{c}\text { Aspectos } \\
\text { fisicos } \\
\text { (reconocimiento } \\
\text { del territorio) }\end{array}$ & $\begin{array}{l}\text { Problemas } \\
\text { ambientales }\end{array}$ & $\begin{array}{c}\text { Actores } \\
\text { presentes }\end{array}$ & Causas & $\begin{array}{r}\text { Responsc } \\
\text { de } \\
\text { solucion }\end{array}$ & $\begin{array}{l}\text { Acciones } \\
\text { para } \\
\text { lo solucionarl }\end{array}$ \\
\hline 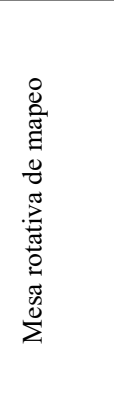 & $\begin{array}{l}\text { Dibujo libre con } \\
\text { plumones o } \\
\text { colores sobre el } \\
\text { mapa para recono- } \\
\text { cer ríos, áreas } \\
\text { verdes, montañas, } \\
\text { áreas naturales } \\
\text { protegidas y } \\
\text { cuerpos de agua }\end{array}$ & $\begin{array}{l}\text { Pegar pequeños } \\
\text { papeles adhesivos } \\
\text { en el lugar donde } \\
\text { se ubica la } \\
\text { problemática } \\
\text { escribiendo cuál } \\
\text { es. Utilizar } \\
\text { sistema de colores } \\
\text { para priorizar } \\
\text { (rojo, amarillo, } \\
\text { verde) }\end{array}$ & $\begin{array}{l}\text { Marcar con } \\
\text { plumones en los } \\
\text { lugares donde } \\
\text { trabajan dichos } \\
\text { actores y } \\
\text { generar leyenda. } \\
\text { Diferenciar } \\
\text { tipos de actores } \\
\text { con sistema de } \\
\text { colores }\end{array}$ & $\begin{array}{l}\text { Rellenar } \\
\text { matriz }\end{array}$ & $\begin{array}{l}\text { Rellenar } \\
\text { matriz }\end{array}$ & $\begin{array}{l}\text { Rellenar } \\
\text { matriz }\end{array}$ \\
\hline 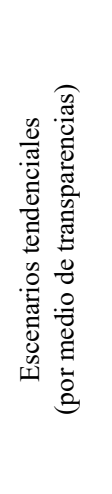 & & $\begin{array}{l}\text { Escenario } \\
\text { negativo: indicar, } \\
\text { si sigue la } \\
\text { tendencia } \\
\text { presente, cuál será } \\
\text { la situación en un } \\
\text { lapso de } 20 \text { años } \\
\text { Escenario } \\
\text { positivo: si se rea- } \\
\text { lizan las acciones } \\
\text { propuestas, cuál } \\
\text { será el escenario } \\
\text { en } 20 \text { años }\end{array}$ & & & & \\
\hline
\end{tabular}

Fuente: elaboración propia.

\section{Fase 3. Generación del SIG}

\section{Digitalización}

Para realizar la digitalización, y reutilizar los mapas bases primeramente se numeraron los puntos para cuidar la correspondencia entre ellos en los diferentes mapas. Posteriormente se generó una capa vectorial de puntos, con los siguientes atributos que de muestran en la Tabla 3.

Los polígonos correspondientes en las sesiones de calle a los elementos perdidos del paisaje, y en los talleres, a los escenarios tendenciales, fueron digitalizados en 
Tabla 3

Sistematización de datos para uso cartográfico

\begin{tabular}{|c|c|c|}
\hline $\begin{array}{l}\text { Nombre } \\
\text { del atributo }\end{array}$ & Tipo & Descripción \\
\hline Clave & Numérica & Número asignado a cada punto \\
\hline Sesión & Texto & Nombre del barrio o colonia; o bien de las organizaciones \\
\hline Problema & Texto & $\begin{array}{l}\text { Problema mencionado por las y los participantes, tal cual } \\
\text { lo escribieron }\end{array}$ \\
\hline Prioridad & Numérica & $\begin{array}{l}\text { Orden de importancia asignado por las y los participantes } \\
\text { a cada problema, siguiendo el método de colores tipo } \\
\text { semáforo de tal manera que } 1 \text { equivalió a lo más } \\
\text { importante (rojo), } 2 \text { a los medianamente importante } \\
\text { (amarillo) y } 3 \text { a lo menos importante (verde) }\end{array}$ \\
\hline Causa & Texto & $\begin{array}{l}\text { Causa identificada por las y los participantes para cada } \\
\text { problema }\end{array}$ \\
\hline $\begin{array}{l}\text { Responsable } \\
\text { gubernamental }\end{array}$ & Texto & $\begin{array}{l}\text { Nivel gubernamental o instituciones identificadas como } \\
\text { responsables de dar solución al problema mencionado }\end{array}$ \\
\hline $\begin{array}{l}\text { Responsable } \\
\text { social }\end{array}$ & Texto & $\begin{array}{l}\text { Responsables no gubernamentales identificados para } \\
\text { poder dar solución al problema mencionado }\end{array}$ \\
\hline $\begin{array}{l}\text { Acción } \\
\text { gubernamental }\end{array}$ & Texto & $\begin{array}{l}\text { Acciones que debería emprender el gobierno para } \\
\text { solucionar el problema, según las y los participantes }\end{array}$ \\
\hline Acción social & Texto & $\begin{array}{l}\text { Acciones que deberían emprender los actores no } \\
\text { gubernamentales para solucionar el problema, según las y } \\
\text { los participantes }\end{array}$ \\
\hline $\begin{array}{c}\text { Actor } \\
\text { presente }\end{array}$ & Texto & $\begin{array}{l}\text { Aplicable únicamente para las sesiones de taller, debían } \\
\text { señalar que actores (instancias, grupos) conocían que ya } \\
\text { estuvieran llevando a cabo acciones para solucionar los } \\
\text { problemas ambientales identificados }\end{array}$ \\
\hline
\end{tabular}

Fuente: elaboración propia.

capas vectoriales de polígono, una por cada sesión o taller y con el único atributo de: uso de suelo. Aquí es importante mencionar que los polígonos no eran exactos, o en algunas partes solamente se colocaron textos o flechas; por lo que fue necesario hacer uso del conocimiento de la ciudad de la autora para definir de manera aproximada las dimensiones de dichos polígonos.

De esta manera al final se tenían 10 capas: una con todos los puntos referentes a los problemas ambientales, una con los polígonos de elementos perdidos del paisaje y ocho de escenarios tendenciales; dos por cada taller. 


\section{Categorización y codificación}

Ésta se hizo buscando primeramente cuáles problemas se repetían, después cuáles menciones diferentes se referían al mismo problema, y así, se crearon categorías generales, que abarcaban a las particulares. La asignación de categorías es una etapa sumamente importante en cualquier SIG, pero también muy delicada cuando se trata de un SIGP, puesto que es un proceso de estandarización, que de alguna manera rompe con la diversidad que es recogida de los conocimientos y percepciones locales; sin embargo, es necesaria para tener una base de datos que sea cartografiable de manera legible. Sieber (2006) menciona al respecto que esta es una de las tensiones reconocidas en los SIGP (P), "la importancia de la particularidad versus la sistematización, en la que el contexto puede ser analizado de forma separada" (p. 495).7 Este mismo proceso se llevó a cabo para los atributos de "responsable" gubernamental y social. Posteriormente y con fines también de hacer el filtrado para la representación cartográfica más ágil; a las diferentes categorías se les asignó una clave numérica o código: por ejemplo contaminación general $=1$, contaminación de agua $=2$. Además, se creó una columna (atributo) denominada "tipo de sesión", en la que le fue asignada la categoría "calle" a las sesiones en los diferentes barrios, "ayuntamiento" a la sesión con el sector gubernamental municipal, y "organización social” a las demás sesiones con ONG. Esta clasificación en la que permitió en los mapas finales contrastar las percepciones de los diferentes tipos de actores de una manera clara a través de la asignación de un color distinto a cada uno.

\section{Representación}

La representación se eligió tomando en cuenta que "para ser efectivo, los mapas y el lenguaje del PPGIS debe comunicar relatos espaciales que clarifiquen y en última instancia politicen los asuntos por los que la gente se siente preocupada" 8 (Weiner, Harris \& Craig, 2002: 9); en este caso, se pretendía mostrar las coincidencias y las diferencias entre las percepciones de los diferentes actores involucrados.

Así, se realizaron los mapas según lugar de afectación —en un primer nivel de clasificación - ; a saber: humedales, ríos, áreas verdes y área habitada. Sin embargo, la categoría "área habitada" englobaba demasiados problemas, por lo que, en un segundo nivel de clasificación, fue dividida por tipo de problema: inundaciones, contaminación, falta de reglamentación o política pública, y falta de intervención territorial.

Para generar cada mapa de puntos, es decir de problemas percibidos, fue necesario duplicar la capa base tres veces (una por actor) y a continuación, para su representa- 
ción, aplicar la herramienta de simbología "basada en reglas", 9 filtrando según los siguientes criterios: el tipo de sesión/actor que lo mencionó, el problema y la importancia que le fue dada. De tal manera que se pudiera lograr que en cada capa solamente se visualizaran los puntos que cumplieran con el tema del mapa y con el actor elegido. Por ejemplo: problema de agua mencionado por actor gubernamental.

Los atributos quedaron representados como se muestra en la Figura 1.

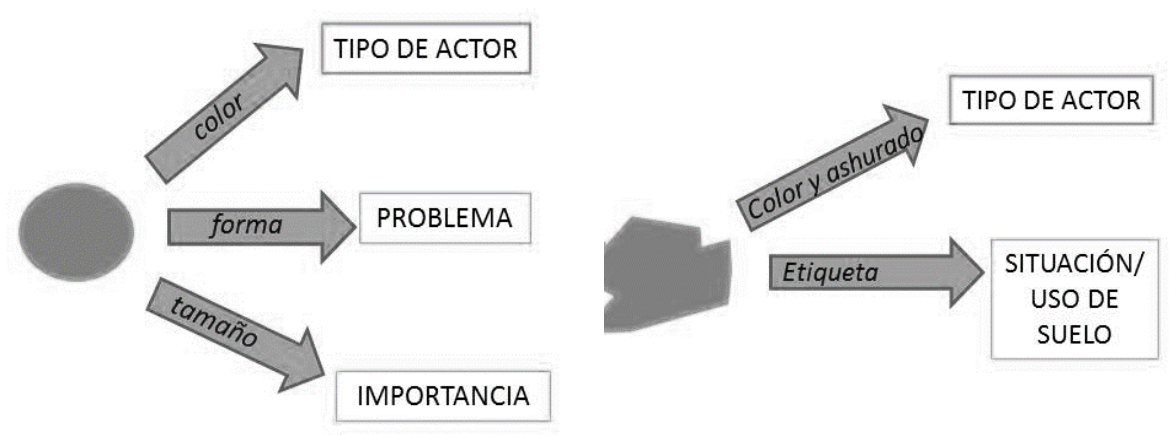

Figura 1. Forma de representación de la información capturada en puntos y polígonos.

Fuente: elaboración propia.

El tipo de relleno utilizado en polígonos fue transparente y achurado - con diferentes patrones de relleno, como líneas o punteado- para facilitar que, aun en el sobrelape de capas se pudieran distinguir los diferentes atributos. En el caso de estos mapas areales, cabe decir que, para no saturar la imagen, también se hizo una selección de las respuestas dadas, por medio de la misma herramienta de "simbología basada en reglas" para poderlas integrar en diferentes mapas según la temática abordada. Así por ejemplo, un mapa muestra únicamente problemas relacionados con el agua, y otro muestra aquellos relacionados con las áreas verdes.

Los mapas generados así posibilitan: contrastar —encontrar los extremos-, comparar -identificar relaciones, diferencias y semejanzas - y complementar la información (los conocimientos y percepciones) entre los diferentes actores. Cumpliendo con la sugerencia de Jordan (1998: 8) de que los "SIGP deben organizar y presentar información pertinente que previamente no estaba disponible, usando las capacidades tecnológicas".

9 Véase en la documentación de QGIS 2.8 en línea el apartado de "clasificación".

https://docs.qgis.org/2.8/es/docs/training_manual/vector_classification/classification.html. 


\section{Complementación}

El potencial de un SIGP no concluye en la mera representación, sino que la utilización de un lenguaje cartográfico convencional y de una plataforma informática, permite combinar datos provenientes del proceso participativo, con cartografía "técnica" u "oficial" derivada de otras fuentes. Con ello se hizo una aproximación a la descripción de community integrated SIG hecha por Weiner \& Harris (2003), quienes afirmaban que éste "provee una arena en la cual diversas formas de conocimiento son integradas dentro de un SIG y en el que los asuntos de políticas de paisaje y manejo de los recursos son desplegados abiertamente" (Weiner \& Harris, 2003: 63). Las posibilidades que ofrece este despliegue son:

- Contextualización: otorgar información complementaria que ayuda a comprender las respuestas dadas por las y los participantes en la cartografía participativa.

- Comparación: establecer semejanzas o diferencias entre lo percibido por los actores, tanto en el presente como en el pasado, ya sea con datos objetivos, o bien con situaciones proyectadas a futuro en planes oficiales.

- Problematización: plantear la existencia de posibles problemas al hacer evidentes las discrepancias entre datos del terreno y las percepciones y conocimientos locales.

Para llevar a cabo estos mapas complementarios se utilizó información edafológica, hidrológica, geomorfológica y de uso de suelo, áreas naturales protegidas, así como las Unidades de Manejo Ambiental (UGAS) del Plan de Ordenamiento Ecológico Territorial municipal.

\section{Fuentes de la información}

En total se realizaron ocho sesiones de mapeo en espacio público, entre el julio y diciembre de 2016. En cada una de ellas participaron en promedio 10 personas, levantándose entre 16 y 30 puntos por sesión. Las y los participantes oscilaron entre los 12 y los 60 años, predominando las personas adultas jóvenes; principalmente mujeres.

En cuanto a los talleres, participaron en ellos las organizaciones sociales: Otros Mundos A.C., Pronatura Sur A.C., Decides A.C., Comité de Cuenca del Valle de Jovel y Consejo Ciudadano. Además, se realizó un taller en el marco del Encuentro Internacional Cuencas y Ciudades Sustentables, organizado por Pronatura Sur A.C. los días 4, 5 y 6 de octubre de 2016. A esta actividad asistieron personas pertenecientes a organizaciones sociales, sector académico, sector gubernamental y sector privado. Finalmente se realizó un taller con 10 integrantes del ayuntamiento municipal 
de San Cristóbal de Las Casas (2015-2018), representantes de la Dirección de Ecología Municipal, Limpia Municipal, Movilidad Urbana, Planeación Territorial, Protección Civil, del departamento de Obras Públicas Municipales, así como del Sistema de Agua Potable y Alcantarillado Municipal.

Así en total fueron cuatro talleres, con una participación de entre cinco y 25 personas, la mayoría adultas jóvenes (entre los 20 y los 35 años aproximadamente), y con una predominancia de mujeres en las ONG, y de hombres en el sector público.

En cuanto a la información cartográfica complementaria utilicé la cartografía base del INEGI (2010), datos sobre Áreas Naturales Protegidas de la CONANP, el Plan de Ordenamiento Ecológico Territorial del Municipio de SCLC (2013), y la propuesta de mejoras a este plan de ordenamiento, realizada por el Instituto para el Desarrollo Sustentable en Mesoamérica A.C. (IDESMAC).

\section{Resultados}

Por motivos de espacio, del total de 25 mapas generados, se muestran aquí únicamente tres ejemplos. En la Figura 2 se da cuenta de la sistematización de percepción de problemas, donde se muestra la complementariedad y contraste entre los tres tipos de actores. En este caso resalta la coincidencia entre actores de identificar la tala de árboles sobre todo en ANP, pero la diferencia en importancia otorgada por ejemplo

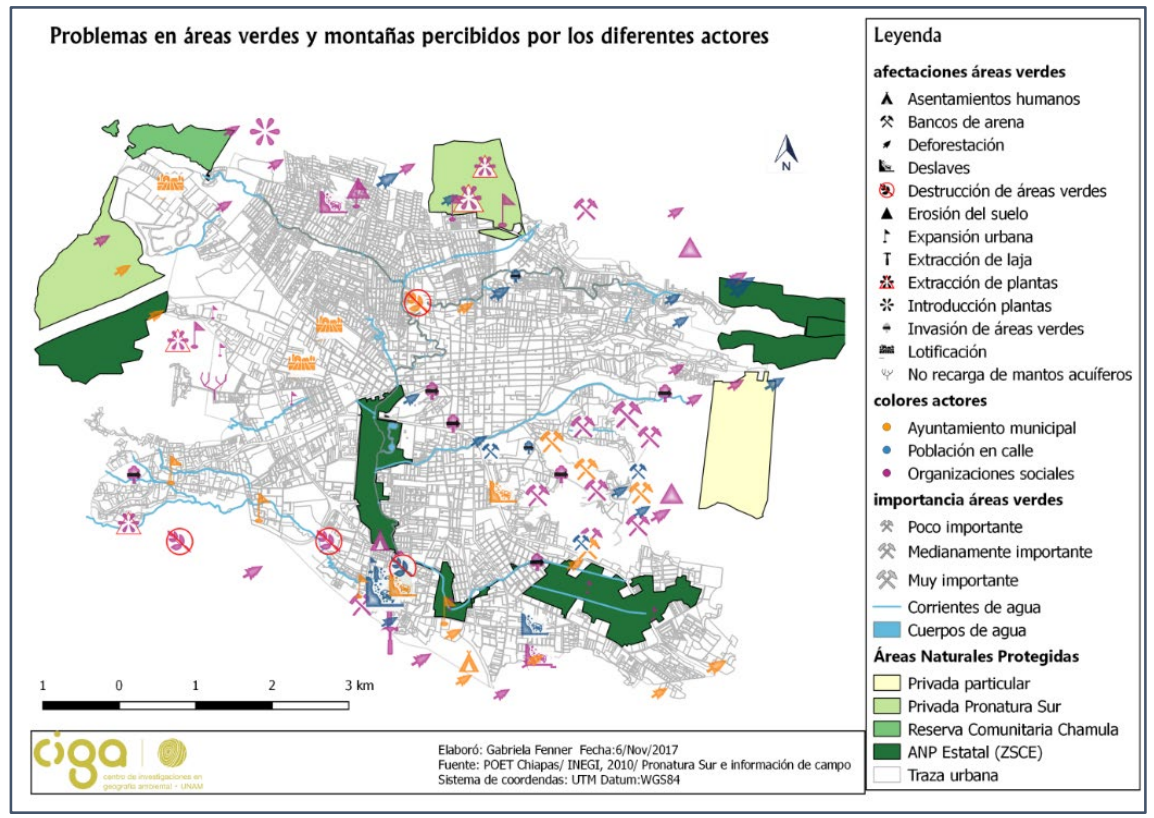

Figura 2. Contrastación de problemas ambientales percibidos por los tres tipos de actores. 


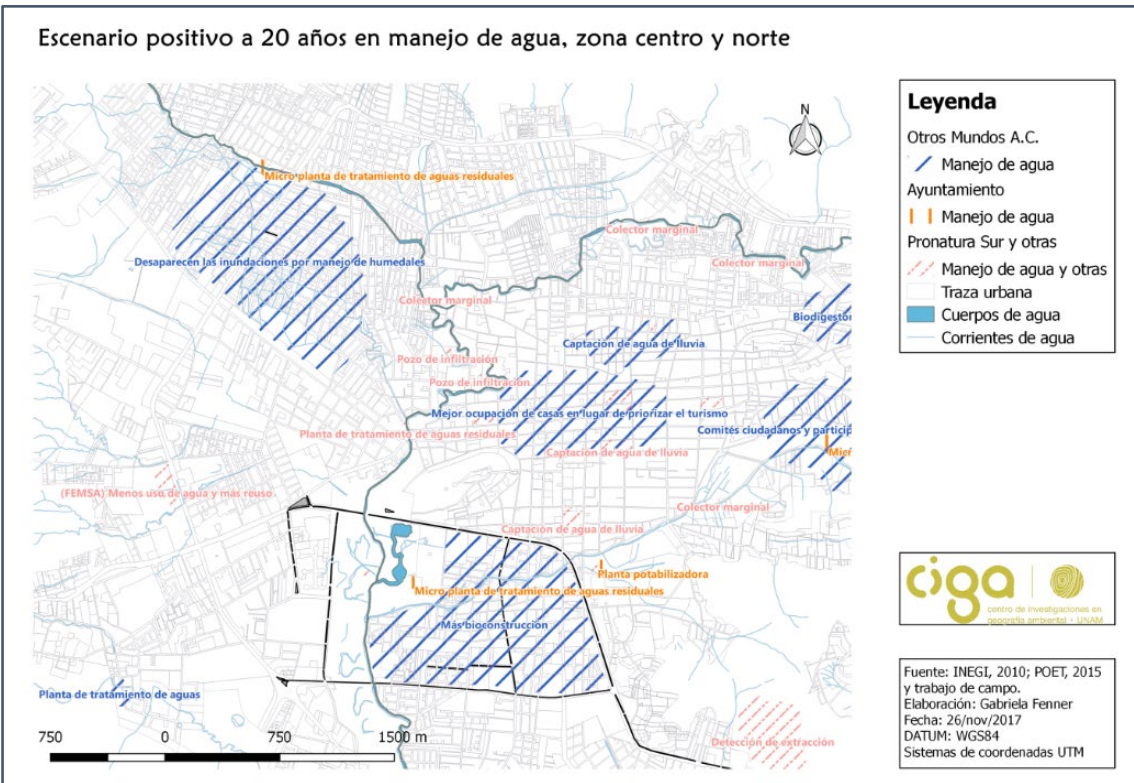

Figura 3. Contrastación escenarios positivos, mostrando posibles sinergias entre actores.

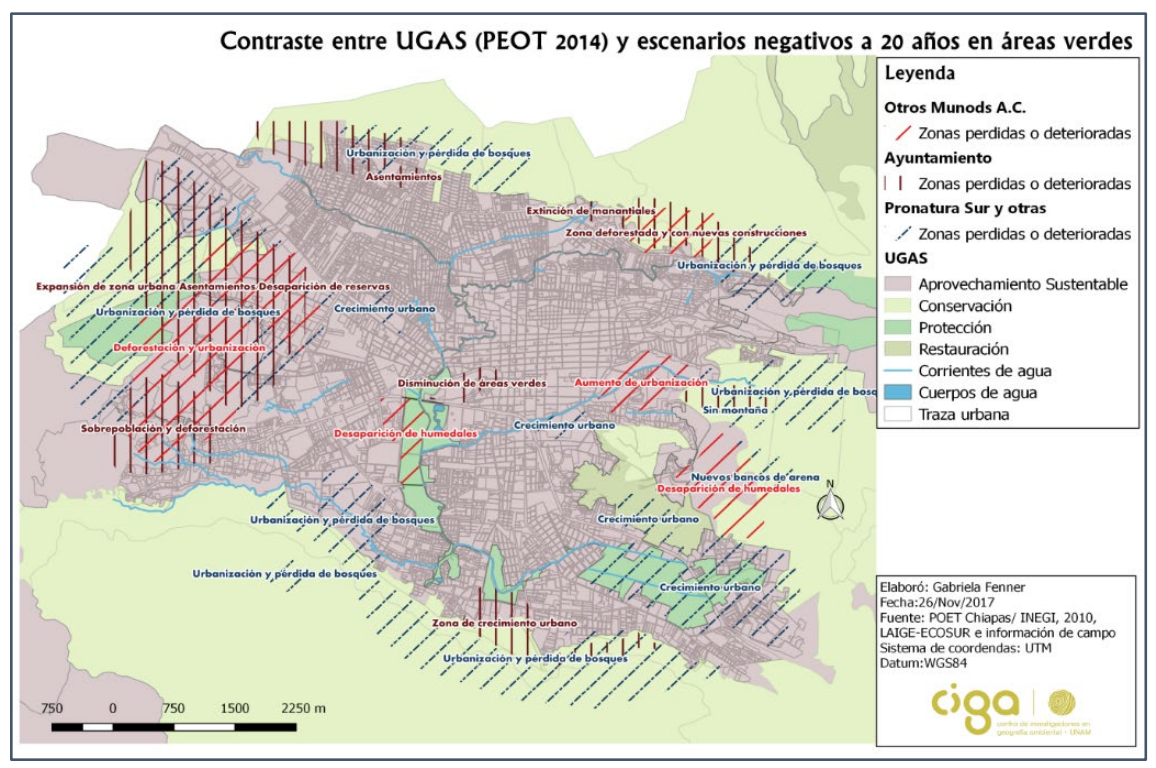

Figura 4. Potencial de los resultados para incidir en la planeación ambiental de la ciudad. 
a la explotación de bancos de arena, donde son las ONG a las que preocupa mayormente. En la Figura 3 se ejemplifica cómo fueron puestos en diálogo los diferentes escenarios tendenciales, en este caso positivos, proyectados por organizaciones sociales y ayuntamiento; visibilizando claramente zonas en cuyo potencial de mejora todos los actores coinciden. En la Figura 4 se muestra el potencial de la metodología al demostrar cómo los escenarios negativos proyectados por los actores pueden ayudar a mejorar el Plan de Ordenamiento Ecológico Territorial Municipal.

\section{Conclusiones e implicaciones prácticas}

En concordancia con el objetivo planteado de este artículo, los comentarios finales hacen una revisión crítica de la metodología en función de los criterios acordados: representatividad, validez, practicidad y aceptabilidad.

- Representatividad: a pesar de que los resultados ayudan a conocer mejor las diferencias y semejanzas entre lo que los actores que participaron perciben como problemas ambientales de la ciudad, son muchos los actores que quedaron fuera y habría que hacer una segunda etapa de ejercicios que buscara integrarlos, de tal manera que se completara mejor la pluralidad de percepciones involucradas. Ésta no es tarea sencilla, pues tal como afirman Hoyt et al. (2005) la habilidad real de los SIGPP de "empoderar a los participantes está determinado en gran medida por el contexto"10 (Hoyt et al., 2005: 1).

- Debido a la relación entre escala local y conocimiento espacial local, las metodologías participativas suelen trabajar dentro de una relación clara: menor escala geográfica, mayor terreno, menor representatividad; por un lado, y mayor escala geográfica, menor terreno, mayor representatividad, por el otro (Carver, 2003). La metodología presentada en esta investigación plantea una posibilidad de combinar estos alcances - y límites - ya que se asume la poca representatividad, al igual que en los ejercicios de IGV, en aras de obtener una visión general de la ciudad, pero a la vez trabaja de manera detallada con diferentes actores. Pues "mientras la densidad del conocimiento especial local, no pueda ser provista de forma apropiada y suficiente por medio de la IGV, la práctica de los SIGP será necesaria para apoyar la planeación local"11 (Verplanke et al., 2016: 7).

- Validez: las limitaciones de representación de los SIG (punto, línea, polígono) condicionan la forma de las respuestas, así como de la cartografía generada; problemas percibidos de manera general tuvieron que ser "reducidos" a puntos concretos. Por el otro lado, el tener que nombrar lugares puntuales llevaba a las y los 
participantes a apelar más a recuerdos de experiencias concretas que a conocimientos abstractos, lo que ratifica la relación positiva entre las percepciones como contenido y la cartografía como herramienta para conocerlas. Es decir que el propósito de la cartografía se reafirma como clave para poder hablar de exactitud y precisión, así como de validez, puesto que una metodología solamente será válida si responde a las necesidades del problema, de tal manera que se consideró más útil poder tener lugares específicos en la ciudad dónde comenzar a actuar, que visualizar físicamente la extensión del problema.

- Practicidad: los mapeos son factibles con poco presupuesto. En términos de instalación, lo único difícil es encontrar, para los puestos de mapeo, lugares públicos transitados, pero que ofrezcan condiciones para detenerse a hacer el ejercicio. Otro aspecto es que, en estos ejercicios al no pedirles a las y los participantes trazar libremente partiendo de una hoja en blanco, pudo salvarse la resistencia que muchas veces se presenta para dibujar (Vajjhala, 2005). Sin embargo, el obstáculo en este caso fue la falta de familiaridad con los mapas, por parte de algunos actores, sobre todo de edad avanzada.

- Aceptabilidad: en general las y los participantes mostraron entusiasmo y disposición por los ejercicios, y algunos manifestaron al final haber aprendido aspectos sobre la ciudad que desconocían, posibilitando cierta expansión del conocimiento espacial local. En el espacio público a veces era más difícil porque la gente no dispone del tiempo o no confía en la utilidad de estos ejercicios. En los espacios de taller, algunos participantes de mayor edad se mostraban más renuentes a usar el mapa, prefiriendo hacerlo de manera oral. Otra cuestión que dificultó la aceptación en algunos actores era que consideraban que la metodología fragmentaba la visión sobre la ciudad y sobre los problemas ambientales, lo que incita a seguir indagando en metodologías más integradoras (Schuurman \& Leszczynski, 2006; Sieber, 2004; Ghose, 2017).

Respecto a estas metodologías participativas que hacen uso de la tecnología es importante también señalar la ausencia de trabajos (al menos en español) en los que se dé cuenta explícitamente del proceso de sistematización y digitalización; es decir de cómo llevar a cabo esa traducción del papel a la computadora perdiendo lo mínimo de información para ser lo más leal posible a la cartografía generada mediante el proceso participativo; lo cual pasa también por una correcta planeación de los ejercicios, en la que de antemano se visualice el tipo de información que se generará y cómo ella formará parte del SIG.

A pesar de lo mucho por mejorar, la investigación permite vislumbrar que metodologías de esta naturaleza otorgan una base sólida de conocimientos, ausencias y percepciones que podría servir como punto de partida para diseñar e implementar programas de educación ambiental, políticas públicas específicas, iniciativas de ley 
en la materia, reglamentos vecinales, entre otros. Es decir, que la misma base puede $\mathrm{y}$, desde mi opinión, debe traducirse en estrategias distintas de educación territorial, otorgando herramientas para el mejor conocimiento del metabolismo de la ciudad, y de los diferentes actores involucrados en la gestión del ambiente urbano.

Siguiendo la clasificación propuesta por Craig y Elwood (1998) respecto a los propósitos del uso de un SIGP en contextos urbanos, tenemos que los resultados de este ejercicio pueden servir a cada una de ellos: administrativos (para conocer en qué zonas atender problemas de inundación por ejemplo), organizacionales (potenciar la coordinación entre organizaciones que comparten objetivos), tácticos (ubicar zonas donde realizar monitores de deforestación), y estratégicos (evaluar impactos del programa de ordenamiento ecológico territorial).

El mapeo participativo y el SIGP "son usados para producir narrativas espaciales (...) que pueden ser adaptadas y revisadas para fundamentar un abanico de proyectos políticos y caracterizaciones de un lugar y sus necesidades" (Elwood, 2006: 704). Y ayudan así a comprender las percepciones sobre los problemas ambientales de quienes habitan el territorio, de quienes con sus necesidades lo afectan, con sus cuerpos lo perciben, con sus hábitos lo cuidan o lo destruyen, con sus conocimientos lo reivindican, y con sus deseos, lo imaginan; así como de quienes, además de ello analizan, gestionan y trabajan para transformarlo.

\section{Bibliografía}

Brown, G. (2012). "Participation GIS (PPGIS) for Regional and Environmental Planning: Reflections on a Decade of Empirical Research", URISA Journal, 25(2): 7-18.

(2016). "A review of sampling effects and responde bias in Internet participatory mapping (PPGIS/PGIS/VGI)", Transactions in GIS, 1-18.

https://doi.org/10.1111/tgis.12207

Carver, S. (2001). "The future of participatory approaches using geographic information: Developing a research agenda for the 21st century", Urban and Regional Information Systems Association (URISA) Journal, vol. 15, APA I, 6172. Recuperado de http://www.urisa.org/Journal/APANo1/carver.pdf

Craig, W.J. and S. Elwood (1998). "How and why community groups use maps and geographic information", Cartography and Geographic Information Systems, 25(2): 95-104. https://doi.org/10.1559/152304098782594616

Elwood, S. (2006). "Critical Issues in Participatory GIS: Deconstructions, Reconstructions and New Research Directions”, Transactions in GIS, 10(5): 693708. https://doi.org/10.1111/j.1467-9671.2006.01023.x

ENDA América Latina-Colombia. (2003). La cartografía social como recurso metodológico para el proyecto Barrios del Mundo. Bogotá, ENDA-Colombia. 
Ghose, R. (2017) "Public-participation GIS", in D. Richardson (ed.), The International Encyclopedia of Geography, Wiley, Nueva York.

Goodchild, M. (2007). "Citizen as sensors: the world of volunteered geography", GeoJournal, 69(4), 211-221. https://doi.org/10.1007/s10708-007-9111-y

Harley, J. (2005). La nueva naturaleza de los mapas: ensayos sobre la historia de la cartografia, 1a. ed., Fondo de Cultura Económica, México.

Hoyt, L.; Khosla, R. \& Canepa, C. (2005). "Leaves, Pebbles, and Chalk: Building a Public Participation GIS in New Delhi, India”, Journal of Urban Technology, 12:1: 1-19. https://doi.org/10.1080/10630730500116479

INEGI (2010). Censo de Población y Vivienda 2010, Instituto Nacional de Estadística y Geografía, Aguascalientes.

INEGI (2015). Conteo Intercensal de Población y Vivienda, Instituto Nacional de Estadística y Geografía, Aguascalientes.

Jankowski, P. (2009). "Towards participatory geographic information systems for community-based environmental decision making", Journal of Environmental Managment, 90(6): 1966-1971. https://doi.org/10.1016/j.jenvman.2007.08.028

Jordan, G. (1998). A public participation GIS for community forestry user groups in Nepal: Putting people before the technology. Proyect Varenius: Empowerment, marginalization, and public participation GIS, Santa Barbara, CA. Recuperado de http://www.negia.ucsb.edu/varenius/ppgis/papers/ jordan.pdf

Kitchin, R. \& Dodge, M. (2007). "Rethinking maps", Progress in Human Goegraphy, 31(3): 331-334. Recuperado de ibliodarq.files.wordpress.com/2013/ 03/kichin-r-y-dodge-m-rethinking-maps.pdf

McCall, M. (2004). "Can participatory-GIS trenghten local-level spatial planning? Suggestions for better practice”, GISDECO, Skudai, Johor, Malaysia, 2-19. Recuperado de

https://www.researchgate.net/publication/228758277 Can participatory-

GIS strengthen local-level spatial planning Suggestions for better practice

McCall, M. (2011). "Mapeando el territorio: paisaje local, conocimiento local, poder local", en G. Bocco, P. Urquijo \& A. Vieyra (edits.), Geografía y ambiente en América Latina, 221-246, Morelia, CIGA-UNAM/INE.

McCall, M. \& Dunn, C. (2012). “Geo-information tools for participatory spatial planning: Fulfilling the criteria for 'good' governance?", Geoforum, 43(1): 81-94. https://doi.org/10.1016/j.geoforum.2011.07.007

Pfeffer, K.; Baud, I.; Denis, E.; Scott, D. \& Sydenstricker-Neto, J. (2010). "Spatial knowledge managment tools in urban development", Assesing and Exploring the state of urban knowledge: ist production, use, and dissemination of the south, oct. 2010, Bruselas. Recuperado de https://hal.archives-ouvertes.fr/hal-00591855

Rambaldi, G.; Chambers, R.; McCall, M. \& Fox, J. (2006). "Practical ethics for PGIS practitioners, facilitators, technology intermediaries and researchers", 
Participatory Learning and Action, 54: 106-113. Recuperado de https://cgspace.cgiar.org/bitstream/handle/10568/76889/14507IIED.pdf?sequenc $\mathrm{e}=1$ \#page $=107$

Riesler, J. y Ares, P. (2013). Manual de mapeo colectivo: recursos cartográficos críticos para procesos territoriales de creación colaborativa, Tinta Limón, Buenos Aires. Recuperado de https:/geoactivismo.org/wp-content/uploads/ 2015/11/Manual_de mapeo_2013.pdf

Rodríguez, G. (2003). Local and popular folklore and culture on hazard and vulnerability meets geographical information system for the risk preparedness of the people of the Barrio San Antonio of Naiguatá, Estado Vargas, Caracas, ProVention/World Bank.

Schlossberg, M. y Shuford, E. (2005). "Delineating "public" and "participation" in PPGIS”, URISA Journal, 16(2): 15-26. Recuperado de https://core.ac.uk/download/pdf/36679952.pdf

Schuurman N. \& Leszczysnki A. (2006). “Ontology-based metadata”, Transactions in GIS, 11(5): 709-26. https://doi.org/10.1111/j.1467-9671.2006.01024.x

Sieber, R. (2004). "Rewiring for a GIS/2", Cartographica, 39(1): 25-39. https://doi.org/10.3138/T6U8-171M-452W-516R

(2006). "Public Participation Geographic Information Systems: A Literature Review and Framework", Annals of the Association of American Geographers, 96(3): 491-507. https://doi.org/10.1111/j.1467-8306.2006.00702.x

SEMARNAT/SEMAHN (2013). Programa de Ordenamiento Ecológico Territorial del Municipio de San Cristóbal de Las Casas (Preliminar), San Cristóbal de Las Casas.

Vajjhala, S. (2005). "Integrating GIS and Paricipatory Mapping in Community Development Planning”, ESRI International User Conference, Sustaintable Development and Humanitarian Affairs, San Diego. Recuperado de https://proceedings.esri.com/library/userconf/proc05/papers/pap1622.pdf

Verplanke, J.; McCall, M.; Uberhuaga, C.; Rambaldi, G. \& Haklay, M. (2016). “A shared perspective for PGIS and VGI", The Cartographic Journal, 53(4): 1-10. https://doi.org/10.1080/00087041.2016.1227552

Weiner, D.; Harris, T. \& Craig, W. (2002). "Community Participation and Geographic Information System", in D. Weiner, T. Harris \& W. Craig, Community Participation and Geographic Information System, Taylor \& Francis, Londres y Nueva York, 3-16.

Weiner, D. \& Harris, T. (2003). "Community-integrated GIS for Land Reform in South Africa”, URISA Journal, 15: 61-73. 